\title{
Ultrasound Assisted Synthesis and Antimicrobial Evaluation of Novel Thiophene Chalcone Derivatives
}

\author{
NARESH PANIGRAHI ${ }^{*}$, SWASTIKA GANGULY ${ }^{2}$, \\ JAGADEESH PANDA $^{3}$ and Y. PRAHARSHA ${ }^{1}$, \\ ${ }^{1}$ GITAM Institute of Pharmacy, GITAM University, Visakhapatnam, India \\ ${ }^{2}$ Dept. of Pharm. Sciences, Birla Institute of Technology, Mesra, India \\ ${ }^{3}$ Raghu College of Pharmacy, Bheemunipatnam, Visakhapatnam, India \\ nareshph28@gmail.com
}

Received 20 February 2014/ Accepted 26 March 2014

\begin{abstract}
A series of 10 novel 5-bromo-thiophene containing chalcone derivatives (SN1-SN10) were synthesized under ultrasonic irradiation in the presence of lithium hydroxide monohydrate $\left(\mathrm{LiOH} . \mathrm{H}_{2} \mathrm{O}\right)$ as a catalyst, which provided the products in good yields after short reaction times under mild conditions. All the synthesized compounds were characterized by spectral data and evaluated for in vitro antibacterial and antifungal activities. Antibacterial and antifungal activities were tested using the agar diffusion method. From the screening studies it was observed that most of the compounds have shown moderate antibacterial and antifungal activities at $500 \mu \mathrm{g} / \mathrm{mL}$ and $100 \mu \mathrm{g} / \mathrm{mL}$ concentrations respectively. Test compounds SN4, SN6, SN7 and SN8 exhibited promising antibacterial activity at $500 \mu \mathrm{g} / \mathrm{mL}$ concentration against the standard ciprofloxacin, whereas all the tested compounds are less active against Candida albicans and moderately active against Aspergillus niger when compared to that of the standard fluconazole.
\end{abstract}

Keywords: Ultrasound irradiation, Conventional method, Lithium hydroxide monohydrate $\left(\mathrm{LiOH} \cdot \mathrm{H}_{2} \mathrm{O}\right)$, 2-Acetyl-5-bromo-thiophene, Aryl aldehydes

\section{Introduction}

Chalcones are $\alpha, \beta$-unsaturated ketones with two aromatic groups bridged by an enone linkage $\left(\mathrm{Ar}-\mathrm{COCH}=\mathrm{CH}-\mathrm{Ar}^{\prime}\right)^{1,2}$. They form the central core for a variety of important biological compounds ${ }^{3}$. They are the intermediates in the synthesis of flavones, biosynthesis of flavonoids, which are substances widespread in plants and with an array of biological activities $^{3}$. Both chalcones and thiophene systems either natural or synthetic are known to exhibit various broad spectrums of pharmacological activities. Thus replacing one of the aryl ring of chalcones with thiophene heterocyclic system has shown an array of biological activities among which antibacterial $^{4,5}$, antifungal ${ }^{4-6}$, anti-inflammatory ${ }^{4,7}$, antihyperlipidemic ${ }^{4}$, anti-oxidant $^{7}$, anti-tuberculosis ${ }^{8}$, anticancer and anti-mitotic ${ }^{7-11}$ ACE inhibitor ${ }^{12}$ activities have been reported. 
Generally chalcones are prepared by aldol condensation between aromatic aldehydes and ketones. This reaction is catalyzed by acids and bases under homogeneous or heterogeneous conditions. Several researchers have reported the synthesis of chalcones by using different bases such as $\mathrm{NaOH}, \mathrm{KOH}, \mathrm{KF}-\mathrm{Al}_{2} \mathrm{O}_{3}, \mathrm{Ba}(\mathrm{OH})_{2}$, hydrotalcites, LiHMDS (Lithium hexa-methyl-disilazide) and calcined $\mathrm{NaNO}_{3} /$ natural phosphate ${ }^{3,13,14}$. The acidcatalysed methodologies include the use of $\mathrm{AlCl}_{3}, \mathrm{BF}_{3}$, dry $\mathrm{HCl}, \mathrm{Zn}(\mathrm{bpy})(\mathrm{OAc})_{2}, \mathrm{TiCl}_{4}$, $\mathrm{Cp}_{2} \mathrm{ZrH}_{2} / \mathrm{NiCl}_{2}$, zeolites and $\mathrm{RuCl}_{3}$. Recently chalcone synthesis has been achieved employing Suzuki reaction ${ }^{1,13-14}$.

Although these methods are suitable for specific synthetic conditions, sometimes, a number of these methods suffer from one or more disadvantages such as long reaction times, expensive reagents, drastic reaction conditions, low yields, tedious work up procedures and co-occurrence of several side reactions. Catalyst recovery and waste disposal problems are again creating environmental issues. As a consequence, the development of simple synthetic routes for widely used organic compounds from readily available reagents is one of the major tasks in organic synthesis ${ }^{3,15}$. In the last few years, the application of ultrasound in synthetic organic chemistry has aroused more and more interest. Sonochemistry offers a more versatile and facile pathway for a large variety of syntheses in comparison to classical methods. Thus, a large number of organic reactions can be carried out in high yield, short reaction time or mild conditions under ultrasound irradiation ${ }^{15}$.

Bhagat et al., ${ }^{13}$ first reported the use of lithium hydroxide monohydrate $\left(\mathrm{LiOH} \cdot \mathrm{H}_{2} \mathrm{O}\right)$ for the synthesis of chalcones under mild conditions which plays the dual role, i.e. generates the enolate from the aryl methyl ketone and activate the aldehyde carbonyl by coordination with $\mathrm{Li}^{+}$.

In the present study we report the synthesis and antimicrobial activities of ten novel 5-bromo-thiophene chalcones using lithium hydroxide monohydrate $\left(\mathrm{LiOH}_{2} \mathrm{H}_{2} \mathrm{O}\right)$ as a catalyst under conventional and ultrasound conditions.

\section{Experimental}

All the aldehydes and the ketone (2-acetyl-5-bromo-thiophene) were purchased from Sigma Aldrich India and Merck India. Lithium hydroxide monohydrate $\left(\mathrm{LiOH} \cdot \mathrm{H}_{2} \mathrm{O}\right)$ was procured from Sisco Research Laboratories (India). All the reagents were used without purification and solvents used were commercial as well as analytical grade.

Melting point was checked using capillary method and was uncorrected. The IR spectra of synthesized compounds were taken on SHIMADZU FTIR spectrophotometer. ${ }^{1}$ HNMR spectra were recorded on Bruker Avance DRX400 (400 MHz, FTNMR) in DMSO, $\mathrm{CDCl}_{3}$ and $\mathrm{MeOD}$ solvents. Chemical shifts were reported as $\delta(\mathrm{ppm})$ relative to TMS as internal standard. Coupling constants $\mathrm{J}$ are expressed in Hertz. Reaction progress was checked by TLC in a solvent-vapor-saturated chamber on glass plates coated with Silica Gel $\mathrm{GF}_{254}$, followed by visualization under UV light $(254 \mathrm{~nm})$. The solvent system used for thin layer and column chromatography was $n$-hexane: ethyl acetate $(8: 2)$.

Sonication was performed using an ultrasonic cleaner (with a frequency of $50 \mathrm{~Hz}$ and a nominal power $170 \mathrm{~W}$ ). The reaction flask was located in the maximum energy area in the cleaner; where the surface of reactants (reaction vessel) is slightly lower than the level of the water.

\section{Synthesis of thiophene-chalcones (Conventional method)}

A mixture of ketone (2-acetyl-5-bromo-thiophene) $(1 \mathrm{mmol})$, corresponding aryl aldehydes $(1 \mathrm{mmol})$, in ethanol $(15 \mathrm{~mL})$ was treated with $\mathrm{LiOH} \cdot \mathrm{H}_{2} \mathrm{O}(4.2 \mathrm{mg}, 0.1 \mathrm{mmol}, 10 \mathrm{~mol} \%)$ 
under magnetically stirred condition (Scheme 1) at room temperature for the period as indicated in Table 1 and kept overnight ${ }^{13}$. The progress of the reaction was monitored by TLC using n-hexane: ethyl acetate $(8: 2)$ as developer. The reaction mixture was poured over crushed ice and acidified with dilute $\mathrm{HCl}$ and extracted with ethyl acetate $(3 \times 5 \mathrm{~mL})$. The combined ethyl acetate extracts were washed with brine solution $(5 \mathrm{~mL})$ and dried over sodium sulphate $\left(\mathrm{Na}_{2} \mathrm{SO}_{4}\right)$. The residue was purified on column chromatography (silica gel with $10 \%$ ethyl acetate in hexane) to afford pure crystals.

\section{Synthesis of thiophene-chalcones (Ultrasound irradiation method)}

A mixture of ketone (2-acetyl-5-bromo-thiophene) $(1 \mathrm{mmol})$, corresponding aryl aldehydes $(1 \mathrm{mmol})$, in ethanol $(5 \mathrm{~mL})$ was treated with $\mathrm{LiOH} \cdot \mathrm{H}_{2} \mathrm{O}(4.2 \mathrm{mg}, 0.1 \mathrm{mmol}, 10 \mathrm{~mol} \%)$ (Scheme 1). The mixture was irradiated in the water bath of an ultrasonic cleaner at the room temperature for the period as indicated in Table 1. The reaction mixture was then poured over crushed ice and acidified with dilute $\mathrm{HCl}$ and extracted with ethyl acetate $(3 \times 5 \mathrm{~mL})$. The combined ethyl acetate extracts were washed with brine solution $(5 \mathrm{~mL})$ and dried over sodium sulphate $\left(\mathrm{Na}_{2} \mathrm{SO}_{4}\right)$. The residue was purified on column chromatography (silica gel with $10 \%$ ethyl acetate in hexane) to afford pure crystals.<smiles>CC(=O)c1ccc(Br)s1</smiles>

2-acetyl-5-bromothiophene

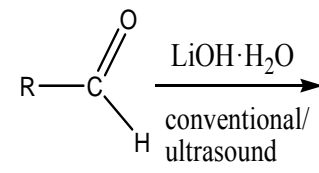

aldehydes<smiles>[R]C=CC(=O)c1ccc(Br)s1</smiles>

Scheme 1. General procedure for synthesis of thiophene-chalcones in presence of $\mathrm{LiOH} \cdot \mathrm{H}_{2} \mathrm{O}$ as a base under conventional and sonochemical methods

Table 1. Physical data of synthesized compounds by conventional and sonochemical methods in the presence of lithium hydroxide monohydrate $\left(\mathrm{LiOH} \cdot \mathrm{H}_{2} \mathrm{O}\right)$ as a base

\begin{tabular}{|c|c|c|c|c|c|c|}
\hline ट्टें & $\mathrm{R}$ & $\begin{array}{l}\text { Reaction Time } \\
\text { (min) under } \\
\text { conventional } \\
\text { method }\end{array}$ & $\begin{array}{c}\% \text { Yield } \\
\text { under } \\
\text { convention } \\
\text { al method } \\
\end{array}$ & $\begin{array}{l}\text { Reaction Time } \\
\text { (min) under } \\
\text { ultrasound } \\
\text { irradiation }\end{array}$ & $\begin{array}{l}\text { \% Yield } \\
\text { under } \\
\text { ultrasound } \\
\text { irradiation } \\
\end{array}$ & $\begin{array}{l}0 \\
0 \\
ن \\
\Sigma\end{array}$ \\
\hline SN1 & & 30 & 83 & 10 & 90 & 102 \\
\hline SN2 & & 30 & 80 & 10 & 93 & 108 \\
\hline SN3 & & 30 & 90 & 10 & 94 & 126 \\
\hline SN4 & & 30 & 78 & 10 & 90 & 98 \\
\hline SN5 & & 30 & 87 & 10 & 95 & 142 \\
\hline
\end{tabular}




\begin{tabular}{|c|c|c|c|c|c|}
\hline SN6 & 30 & 82 & 10 & 93 & 129 \\
\hline SN7 & 30 & 85 & 10 & 91 & 138 \\
\hline SN8 & 30 & 88 & 10 & 94 & 149 \\
\hline SN9 & 30 & 77 & 10 & 91 & 147 \\
\hline SN10 & 30 & 83 & 10 & 94 & 91 \\
\hline
\end{tabular}

\section{Results and Discussion}

The products were characterized by the spectral data of ${ }^{1} \mathrm{H}$ NMR, IR and elemental analysis.

\section{Spectral data of the synthesized compounds}

\section{Compound SN1}

(E)-3-(4-Bromophenyl)-1-(5-bromothiophen-2-yl)-prop-2-en-1-one: White crystalline needles; m.p: $102{ }^{\circ} \mathrm{C}$; IR $(\mathrm{KBr})\left(\mathrm{cm}^{-1}\right)$ : $1711.17(\mathrm{C}=\mathrm{O}), 1598.61(\mathrm{C}=\mathrm{C}), 659.74(\mathrm{C}-\mathrm{S})$, $532.64(\mathrm{C}-\mathrm{Br}) .{ }^{1} \mathrm{H}$ NMR $\left(400 \mathrm{MHz} ; \mathrm{CDCl}_{3}\right): \delta(\mathrm{ppm}): \delta 7.77(\mathrm{~d}, \mathrm{~J}=7.3 \mathrm{~Hz}, 1 \mathrm{H}), 7.59(\mathrm{~d}, \mathrm{~J}=$ $17.0 \mathrm{~Hz}, 1 \mathrm{H}), 7.564(\mathrm{~d}, \mathrm{~J}=7.5 \mathrm{~Hz} 2 \mathrm{H}), 7.49(\mathrm{~d}, \mathrm{~J}=6.3 \mathrm{~Hz}, 2 \mathrm{H}), 7.164(\mathrm{~d}, \mathrm{~J}=7.3 \mathrm{~Hz}, 1 \mathrm{H})$, $6.84(\mathrm{~d}, \mathrm{~J}=17.0 \mathrm{~Hz}, 1 \mathrm{H})$. Anal. Calcd for $\mathrm{C}_{13} \mathrm{H}_{8} \mathrm{Br}_{2} \mathrm{OS}$ : C, 41.97; H, 2.17; Br, 42.95; O, 4.30; S, 8.62. Found: C, 41.78; H, 2.10; Br, 42.93; O, 4.28; S, 8.60.

\section{Compound SN2}

(E)-3-(2-Bromophenyl)-1-(5-bromothiophen-2-yl) - prop-2-en-1-one: White crystals; m.p: $108{ }^{\circ} \mathrm{C}$; IR (KBr) $\left(\mathrm{cm}^{-1}\right): 1701.56(\mathrm{C}=\mathrm{O}), 1599.13(\mathrm{C}=\mathrm{C}), 676.42(\mathrm{C}-\mathrm{S}), 578.96(\mathrm{C}-\mathrm{Br}) .{ }^{1} \mathrm{H}$ NMR (400 MHz; $\left.\mathrm{CDCl}_{3}\right): \delta(\mathrm{ppm}): \delta 8.418(\mathrm{~d}, \mathrm{~J}=8.5 \mathrm{~Hz}, 2 \mathrm{H}), 8.200(\mathrm{~d}, \mathrm{~J}=8.5 \mathrm{~Hz}, 2 \mathrm{H})$, $7.607(\mathrm{~d}, \mathrm{~J}=17.0 \mathrm{~Hz}, 1 \mathrm{H}), 7.593(\mathrm{~d}, \mathrm{~J}=6.3 \mathrm{~Hz} 1 \mathrm{H}), 7.263(\mathrm{~d}, \mathrm{~J}=17.0 \mathrm{~Hz}, 1 \mathrm{H}), 7.158(\mathrm{~m}$, 1H). Anal. Calcd for $\mathrm{C}_{13} \mathrm{H}_{8} \mathrm{Br}_{2} \mathrm{OS}$ : C, 41.96; H, 2.17; Br, 42.95; O, 4.30; S, 8.62. Found: $\mathrm{C}$, 41.81; H, 2.10; Br, 42.90; O, 4.28; S, 8.60.

\section{Compound SN3}

(E)-1-(5-Bromothiophen-2-yl)-3-(4-(dimethylamino) phenyl) prop-2-en-1-one: Orange needles; m.p:126 ${ }^{\circ} \mathrm{C}$; IR $(\mathrm{KBr})\left(\mathrm{cm}^{-1}\right): 1728.24(\mathrm{C}=\mathrm{O}), 1568.89(\mathrm{C}=\mathrm{C}), 1189.71(\mathrm{C}-\mathrm{N}), 538.42$ (C-Br). $\quad{ }^{1} \mathrm{H}$ NMR (400 MHz; $\left.\mathrm{CDCl}_{3}\right): \delta(\mathrm{ppm}): \delta 7.843(\mathrm{~d}, \mathrm{~J}=17.0 \mathrm{~Hz}, 1 \mathrm{H}), 7.67 \mathrm{~d}, \mathrm{~J}=6.3$ $\mathrm{Hz}, 1 \mathrm{H}), 7.567$ (d, J = 8.5 Hz, 2H), $7.262(\mathrm{~d}, \mathrm{~J}=17.0 \mathrm{~Hz} 1 \mathrm{H}), 6.706(\mathrm{~d}, \mathrm{~J}=8.5 \mathrm{~Hz}, 2 \mathrm{H}), 6.676$ (m, 1H), 3.055 (s, 6H). Anal. Calcd for $\mathrm{C}_{15} \mathrm{H}_{14} \mathrm{BrNOS}$ : C, 53.58; H, 4.20; Br, 23.76; N, 4.17; O, 4.76; S, 9.53. Found: C, 53.60; H, 4.22; Br, 23.74; N, 4.15; O, 4.75; S, 9.56.

\section{Compound SN4}

(E)-1-(5-Bromothiophen-2-yl)-3-(2-hydroxyphenyl) prop-2-en-1-one: Green crystals; m.p: $98{ }^{\circ} \mathrm{C}$; IR $(\mathrm{KBr})$ in $\mathrm{cm}^{-1}: 3410.14(-\mathrm{OH}$ stretch), $1669.25(\mathrm{C}=\mathrm{O}), 1542.87(\mathrm{C}=\mathrm{C}), 1015.82$ (C-Br). ${ }^{1} \mathrm{H}$ NMR (400 MHz; $\left.\mathrm{CDCl}_{3}\right): \delta(\mathrm{ppm}): \delta 8.073$ (d, J = 15.6 Hz, 1H), $7.582(\mathrm{dd}, \mathrm{J}=7.4$, 
$1.5 \mathrm{~Hz}, 2 \mathrm{H}), 7.499(\mathrm{~d}, \mathrm{~J}=7.5 \mathrm{~Hz}, 1 \mathrm{H}), 7.29(\mathrm{~d}, 1 \mathrm{H}), 6.99(\mathrm{~d}, 1 \mathrm{H}), 7.147$ (d, J = $7.3 \mathrm{~Hz}, 1 \mathrm{H})$, $6.839(\mathrm{~d}, \mathrm{~J}=15.6 \mathrm{~Hz}, 1 \mathrm{H}), 5.528(\mathrm{~s}, 1 \mathrm{H})$. Anal. Calcd for $\mathrm{C}_{13} \mathrm{H}_{9} \mathrm{BrO}_{2} \mathrm{~S}: \mathrm{C}, 50.50 ; \mathrm{H}, 2.93 ; \mathrm{Br}$, 25.84; O, 10.35; S, 10.37. Found: C, 50.47; H, 2.96; Br, 25.85; O, 10.38; S, 10.33 .

\section{Compound SN5}

(E)-1-(5-Bromothiophen-2-yl)-3-(4-methoxyphenyl) prop-2-en-1-one: Yellow crystals; m.p: $142{ }^{\circ} \mathrm{C}$; IR $(\mathrm{KBr})$ in $\mathrm{cm}^{-1}: 1677.10(\mathrm{C}=\mathrm{O}), 1597.13(\mathrm{C}=\mathrm{C}), 1069.88(\mathrm{C}-\mathrm{O}), 608.31(\mathrm{C}-\mathrm{Br})$. ${ }^{1} \mathrm{H}$ NMR $\left(400 \mathrm{MHz} ; \mathrm{CDCl}_{3}\right): \delta(\mathrm{ppm}): \delta 8.00-7.94(\mathrm{~m}, 2 \mathrm{H}), 7.67(\mathrm{~d}, \mathrm{~J}=17.0 \mathrm{~Hz}, 1 \mathrm{H}), 7.51$ $(\mathrm{d}, \mathrm{J}=7.5 \mathrm{~Hz}, 1 \mathrm{H}), 7.30-7.24(\mathrm{~m}, 2 \mathrm{H}), 7.01(\mathrm{~d}, \mathrm{~J}=7.3 \mathrm{~Hz}, 1 \mathrm{H}), 6.70(\mathrm{~d}, \mathrm{~J}=17.0 \mathrm{~Hz}, 1 \mathrm{H})$, 3.80 (s, 3H). Anal. Calcd for: $\mathrm{C}_{14} \mathrm{H}_{11} \mathrm{BrO}_{2} \mathrm{~S}$ : C, 52.03; H, 3.43; Br, 24.72; O, 9.90; S, 9.92. Found: C, 52.13; H, 3.40; Br, 24.67; O, 9.92; S, 9.95.

\section{Compound SN6}

(E)-1-(5-Bromothiophen-2-yl)-3-(3, 4-dimethoxyphenyl) prop-2-en-1-one: Yellow crystals; m.p: $129^{\circ} \mathrm{C}$; IR $(\mathrm{KBr})$ in $\mathrm{cm}^{-1}$ : $1658.40(\mathrm{C}=\mathrm{O}), 1607.83(\mathrm{C}=\mathrm{C}), 1076.28(\mathrm{C}-\mathrm{O}), 687.08(\mathrm{C}-\mathrm{Br})$. ${ }^{1} \mathrm{H}$ NMR (400 MHz; $\left.\mathrm{CDCl}_{3}\right): \delta(\mathrm{ppm}): \delta 7.782(\mathrm{~d}, \mathrm{~J}=17.0 \mathrm{~Hz}, 1 \mathrm{H}), 7.762(\mathrm{~d}, \mathrm{~J}=7.5 \mathrm{~Hz}$, 2H), $7.40(\mathrm{~d}, \mathrm{~J}=7.5 \mathrm{~Hz}, 2 \mathrm{H}), 7.597(\mathrm{~s}, 1 \mathrm{H}), 6.92(\mathrm{~d}, \mathrm{~J}=17.0 \mathrm{~Hz}, 1 \mathrm{H}), 3.95(\mathrm{~s}, 6 \mathrm{H})$. Anal. Calcd for: $\mathrm{C}_{15} \mathrm{H}_{13} \mathrm{BrO}_{3} \mathrm{~S}$ : C, 51.01; H, 3.71; Br, 22.62; O, 13.59; S, 9.08. Found: C, 51.09; H, $3.65 ; \mathrm{Br}, 22.67$; O, 13.55; S, 9.18.

\section{Compound SN7}

(E)-1-(5-Bromothiophen-2-yl)-3-(4-hydroxy-3-methoxyphenyl) prop-2-en-1-one: Yellow crystals; m.p: $138{ }^{\circ} \mathrm{C}$; IR (KBr) in $\mathrm{cm}^{-1}: 3474.16(-\mathrm{OH}$ stretch), $1689.25(\mathrm{C}=\mathrm{O}), 1595.76$ $(\mathrm{C}=\mathrm{C}), 1017.92(\mathrm{C}-\mathrm{O}) .{ }^{1} \mathrm{H}$ NMR $\left(400 \mathrm{MHz}, \mathrm{CDCl}_{3}\right): \delta(\mathrm{ppm}): \delta 7.67(\mathrm{~d}, \mathrm{~J}=15.6 \mathrm{~Hz}, 1 \mathrm{H})$, $7.51(\mathrm{~d}, \mathrm{~J}=7.5 \mathrm{~Hz}, 1 \mathrm{H}), 7.40(\mathrm{~d}, \mathrm{~J}=1.5 \mathrm{~Hz}, 1 \mathrm{H}), 7.20(\mathrm{~d}, \mathrm{~J}=7.5 \mathrm{~Hz}, 1 \mathrm{H}), 7.08(\mathrm{dd}, \mathrm{J}=7.5$, $1.5 \mathrm{~Hz}, 1 \mathrm{H}), 7.01(\mathrm{~d}, \mathrm{~J}=7.3 \mathrm{~Hz}, 1 \mathrm{H}), 6.70(\mathrm{~d}, \mathrm{~J}=15.6 \mathrm{~Hz}, 1 \mathrm{H}), 6.00(\mathrm{~s}, 1 \mathrm{H}), 3.84(\mathrm{~s}, 3 \mathrm{H})$. Anal. Calcd for: $\mathrm{C}_{14} \mathrm{H}_{11} \mathrm{BrO}_{3} \mathrm{~S}$ : C, 49.57; H, 3.27; Br, 23.56; O, 14.15; S, 9.45. Found: $\mathrm{C}$, 49.57; H, 3.28; Br, 23.50; O, 14.20; S, 9.47.

\section{Compound $S N 8$}

(E)-1-(5-Bromothiophen-2-yl)-3-(p-tolyl)prop-2-en-1-one: Yellow crystals; m.p: $149^{\circ} \mathrm{C}$; IR $(\mathrm{KBr})$ in $\mathrm{cm}^{-1}: 1647.90(\mathrm{C}=\mathrm{O}), 1590.18(\mathrm{C}=\mathrm{C}), 689.71(\mathrm{C}-\mathrm{Br}) .{ }^{1} \mathrm{H}$ NMR $\left(400 \mathrm{MHz} ; \mathrm{CDCl}_{3}\right): \delta$ (ppm): $\delta 7.596(\mathrm{~d}, \mathrm{~J}=17.0 \mathrm{~Hz}, 1 \mathrm{H}), 7.583(\mathrm{~d}, \mathrm{~J}=6.3 \mathrm{~Hz} 1 \mathrm{H}), 7.545(\mathrm{~d}, \mathrm{~J}=9.0 \mathrm{~Hz}, 2 \mathrm{H}), 7.140$ $(\mathrm{m}, 1 \mathrm{H}), 7.251(\mathrm{~d}, \mathrm{~J}=9.0 \mathrm{~Hz}, 2 \mathrm{H}), 2.396(\mathrm{~s}, 3 \mathrm{H})$. Anal. Calcd for: $\mathrm{C}_{14} \mathrm{H}_{11} \mathrm{BrOS}: \mathrm{C}, 54.74 ; \mathrm{H}$, 3.61; Br, 26.01; O, 5.21; S, 10.44. Found: C, 54.70; H, 3.65; Br, 26.05; O, 5.19; S, 10.40.

\section{Compound SN9}

(E)-1-(5-Bromothiophen-2-yl)-3-(4-chlorophenyl)prop-2-en-1-one: Yellow crystals; m.p: $147^{\circ} \mathrm{C}$; IR $(\mathrm{KBr})$ in $\mathrm{cm}^{-1}: 1648.77(\mathrm{C}=\mathrm{O}), 1589.35(\mathrm{C}=\mathrm{C}), 843.60(\mathrm{C}-\mathrm{Cl}) .{ }^{1} \mathrm{H}$ NMR $(400$ $\left.\mathrm{MHz} ; \mathrm{CDCl}_{3}\right): \delta(\mathrm{ppm}): \delta 7.761(\mathrm{~d}, \mathrm{~J}=9.0 \mathrm{~Hz}, 2 \mathrm{H}), 7.591(\mathrm{~d}, \mathrm{~J}=6.3 \mathrm{~Hz}, 1 \mathrm{H}), 7.583(\mathrm{~d}, \mathrm{~J}=$ $17.0 \mathrm{~Hz}, 1 \mathrm{H}), 7.414(\mathrm{~d}, \mathrm{~J}=9.0 \mathrm{~Hz}, 2 \mathrm{H}), 7.263(\mathrm{~d}, \mathrm{~J}=17.0 \mathrm{~Hz}, 1 \mathrm{H}), 7.158(\mathrm{~m}, 1 \mathrm{H})$. Anal. Calcd for: $\mathrm{C}_{13} \mathrm{H}_{8} \mathrm{BrClOS}$ : C, 47.66; H, 2.46; Br, 24.39; Cl, 10.82; O, 4.88; S, 9.79. Found: C, 47.60; H, 2.43; Br, 24.40; Cl, 10.85; O, 4.90; S, 9.82.

\section{Compound SN10}

(E)-3-(Anthracen-9-yl)-1-(5-bromothiophen-2-yl) prop-2-en-1-one: Reddish brown crystals; m.p: $91^{\circ} \mathrm{C}$; IR $(\mathrm{KBr})$ in $\mathrm{cm}^{-1}$ : 3030.52 (aromatic C-H- stretch), $1710.79(\mathrm{C}=\mathrm{O}), 1575.80$ $(\mathrm{C}=\mathrm{C}), 1065.28(\mathrm{C}-\mathrm{Br}) .{ }^{1} \mathrm{H}$ NMR $\left(400 \mathrm{MHz} ; \mathrm{CDCl}_{3}\right): \delta(\mathrm{ppm}): \delta 8.413(\mathrm{~s}, 1 \mathrm{H}), 8.045(\mathrm{t}, \mathrm{J}$ $=1.5 \mathrm{~Hz}, 2 \mathrm{H}), 7.99(\mathrm{t}, \mathrm{J}=1.9 \mathrm{~Hz}, 2 \mathrm{H}), 7.86(\mathrm{~d}, \mathrm{~J}=17.0 \mathrm{~Hz}, 1 \mathrm{H}), 7.434-7.458(\mathrm{~m}, 4 \mathrm{H}), 7.389$ 
$(\mathrm{d}, \mathrm{J}=7.1 \mathrm{~Hz}, 1 \mathrm{H}), 7.345(\mathrm{~d}, \mathrm{~J}=7.1 \mathrm{~Hz}, 1 \mathrm{H}), 6.938(\mathrm{~d}, \mathrm{~J}=17.0 \mathrm{~Hz}, 1 \mathrm{H})$. Anal. Calcd for: $\mathrm{C}_{21} \mathrm{H}_{13} \mathrm{BrOS}$ : C, 64.13; H, 3.33; Br, 20.32; O, 4.07; S, 8.15. Found: C, 64.10; H, 3.36; Br, 20.28; O, 4.10; S, 8.18.

\section{Antimicrobial screening}

The in vitro antibacterial and antifungal activities of thiophene chalcones were determined using agar diffusion method ${ }^{16,17}$.

\section{Antibacterial activity}

Four different bacterial test organisms such as two gram-positive bacteria Bacillus substills (NCIM 2699), Staphylococcus aureus (NCIM 2672) and two gram-negative bacteria Escherichia coli (NCIM 2685) and Pseudomonas aeruginosa (NCIM 5029) were obtained from NCIM (National Collection of Industrial Microorganisms), Pune, India. Cultures of test organisms were maintained on nutrient agar slants and were sub cultured in Petri dishes prior to activity. Ciprofloxacin was used as standard drug for the comparison of antibacterial activity. Stock solutions of synthesized chalcones were diluted in $1 \%$ dimethyl sulfoxide (DMSO) to give a final concentration ranging from 100 to $500 \mu \mathrm{g} / \mathrm{mL}$ for determining the zone of inhibition values. Using a sterile borer, wells of $6 \mathrm{~mm}$ diameter were made and each well was labeled. The stock solutions at concentrations $(100 \mu \mathrm{g} / \mathrm{mL}, 250 \mu \mathrm{g} / \mathrm{mL}$ and $500 \mu \mathrm{g} / \mathrm{mL})$ was added to respective wells aseptically and labeled accordingly. The plates were kept undisturbed for $1 \mathrm{~h}$ at room temperature to allow the diffusion of the solution properly in the nutrient agar medium and then transferred into the incubator. After incubation of the plates at $37 \pm 1{ }^{\circ} \mathrm{C}$ for $24 \mathrm{~h}$, the diameter of zone inhibition surrounding each of the discs was measured with the help of an antibiotic zone reader. Simultaneously, controls were maintained employing $0.1 \mathrm{~mL}$ of DMSO to observe the solvent effects and the results were represented in Table 2 .

Table 2. Antibacterial activity of thiophene-chalcone derivatives in terms of zone of inhibition $(\mathrm{mm})$

\begin{tabular}{|c|c|c|c|c|}
\hline \multirow{3}{*}{ Compd. } & \multicolumn{4}{|c|}{ Diameter of zone of inhibition, $\mathrm{mm}^{\mathrm{a}}$ at $500 \mu \mathrm{g} / \mathrm{mL}$ concentration. } \\
\hline & \multicolumn{2}{|c|}{ Gram-positive bacteria } & \multicolumn{2}{|c|}{ Gram-negative bacteria } \\
\hline & $\begin{array}{c}\text { Bacillus } \\
\text { substills } \\
\text { (NCIM 2699) }\end{array}$ & $\begin{array}{c}\text { Staphylococcus } \\
\text { aureus } \\
\text { (NCIM 2672) }^{\mathrm{b}}\end{array}$ & $\begin{array}{l}\text { Escherichia coli } \\
\text { (NCIM 2685) }^{\mathrm{b}}\end{array}$ & $\begin{array}{l}\text { Pseudomonas } \\
\text { aeruginosa } \\
\text { (NCIM 5029) }^{\mathrm{b}}\end{array}$ \\
\hline SN1 & 16 & 18 & 20 & 15 \\
\hline SN2 & 15 & 15 & 18 & 18 \\
\hline SN3 & 22 & 18 & 16 & 12 \\
\hline SN4 & 19 & 18 & 24 & 20 \\
\hline SN5 & 17 & 22 & 19 & 17 \\
\hline SN6 & 20 & 17 & 22 & 20 \\
\hline SN7 & 24 & 21 & 13 & 18 \\
\hline SN8 & 22 & 23 & 18 & 14 \\
\hline SN9 & 10 & 11 & 15 & 13 \\
\hline SN10 & 15 & 17 & 12 & 11 \\
\hline Ciprofloxacin $^{\mathrm{c}}$ & 26 & 27 & 28 & 25 \\
\hline $\mathrm{DMSO}^{\mathrm{c}}$ & - & - & - & - \\
\hline
\end{tabular}

${ }^{a} 25-40 \mathrm{~mm}$ significantly active, $12-25 \mathrm{~mm}$ moderately active, $<12$ less active. ${ }^{b}$ Data represent the mean of three replicates. ${ }^{c}$ Positive control (standard): ciprofloxacin and negative control (DMSO). -Indicates no activity 


\section{Antifungal activity}

Antifungal activity was also done by agar diffusion method. To carry out the antifungal activity, the test organisms Candida albicans (NCIM 3466) and Aspergillus niger (NCIM 548) strains were procured from NCIM (National Collection of Industrial Microorganisms), pune, India. The antifungal activity of synthesized compounds was determined by observing the zone of inhibition in comparison to the standard antifungal fluconazole.

Test compounds were dissolved in DMSO to make a stock solution of $1 \mathrm{mg} / \mathrm{mL}$. The fresh sub culture of strains in normal saline was added to the sterile assay medium (Saboraud Dextrose agar) at $40-45^{\circ} \mathrm{C}$ and mixed well. The medium was poured into each of the petridishes. Using a sterile borer, wells of $6 \mathrm{~mm}$ diameter were made and each well was labeled. DMSO was kept as negative control. The stock solution at concentration $100 \mu \mathrm{g} / \mathrm{mL}$ was added to respective wells aseptically and labeled accordingly and the media was allowed to stand for $5 \mathrm{~min}$. The petridishes were kept aside for $1 \mathrm{~h}$ and then incubated at $25^{\circ} \mathrm{C}$ for $48 \mathrm{~h}$ at inverted position. Zone of inhibition was measured and the average of the three readings was calculated and the results were represented in Table 3.

Table 3. Antifungal activity of thiophene-chalcone derivatives in terms of zone of inhibition $(\mathrm{mm})$

\begin{tabular}{|c|c|c|}
\hline \multirow[b]{2}{*}{ Compound } & \multicolumn{2}{|c|}{ Diameter of zone of inhibition, $\mathrm{mm}^{\mathrm{a}}$ at $100 \mu \mathrm{g} / \mathrm{mL}$ concentration. } \\
\hline & $\begin{array}{l}\text { Candida albicans } \\
\text { (NCIM 3466) }^{\mathrm{b}}\end{array}$ & Aspergillus niger (NCIM 548) \\
\hline SN1 & 7 & 10 \\
\hline SN2 & 8 & 11 \\
\hline SN3 & 6 & 10 \\
\hline SN4 & 11 & 12 \\
\hline SN5 & 10 & 12 \\
\hline SN6 & 11 & 12 \\
\hline SN7 & 8 & 11 \\
\hline SN8 & 4 & 6 \\
\hline SN9 & 8 & 9 \\
\hline SN10 & 6 & 8 \\
\hline Fluconazole $^{\mathrm{c}}$ & 18 & 20 \\
\hline $\mathrm{DMSO}^{\mathrm{c}}$ & - & - \\
\hline
\end{tabular}

${ }^{a} 15-20 \mathrm{~mm}$ significantly active, $10-15 \mathrm{~mm}$ moderately active, $<10$ less active. ${ }^{b}$ Data represent the mean of three replicates. ${ }^{c}$ Positive control (standard): fluconazole and negative control (DMSO). Indicates no activity

In the present study, a series of 10 novel 5-bromo-thiophene containing chalcone derivatives (SN1-SN10) were synthesized by Claisen-Schmidt condensation of 2-acetyl-5bromo-thiophene (ketone) with different aromatic aldehydes under conventional and ultrasound conditions in the presence of lithium hydroxide monohydrate $\left(\mathrm{LiOH}_{2} \mathrm{H}_{2} \mathrm{O}\right)$ as a catalyst .

At first to investigate the role of ultrasonic irradiation in this method, the reaction was carried out in the presence of lithium hydroxide monohydrate $\left(\mathrm{LiOH} \mathrm{H}_{2} \mathrm{O}\right)$ as a catalyst under stirring condition (conventional method of synthesis) in ethanol at room temperature and the effects on reaction time and percentage yield were compared with the results of sonication method (Scheme 1). The results are summarized in Table1. Excellent results were obtained employing LiOH. $\mathrm{H}_{2} \mathrm{O}$ as a base. The reactions were carried out in short times i.e. 
less than 30 min under conventional method of synthesis and in case of sonication method it was less than $10 \mathrm{~min}$. This is due to the impact of acoustic energy which was evident in reduction of the reaction time by inducing rapid collisions between the molecules and resulting faster reaction (also possibly due to the formation of local hot spots) to form chalcones. In case of sonochemically assisted synthesis the yield of the thiophene chalcones were almost increased when it is compared to conventionally synthesized chalcone (Table 1). In general, the reactions were clean and the isolated products were obtained in pure form without further purification by column chromatography and formation of crystals was rapid. The chalcone formation took place with excellent chemoselectivity since most of the chalcones formed were trans configuration $(J=15$ to $17.0 \mathrm{~Hz})$. Since, the objective of the work was to find out the effect of ultrasound at normal room temperature (mild conditions). The temperature was kept constant throughout the reaction.

All synthesized novel thiophene chalcone derivatives (SN1-SN10) were screened for their antibacterial activity by using the agar-diffusion method, against a panel of selected gram-positive and gram-negative bacteria's. The investigation of antibacterial screening data revealed that all the tested compounds SN1 to SN10 showed moderate bacterial inhibition. The results were summarized in Table 2, suggest that only the compounds SN9 and SN10 are less active against the tested compounds, but all other compounds show moderate antimicrobial activity. Compounds SN7 and SN8 showed highest zone of inhibition (24 mm and $23 \mathrm{~mm}$ ) against gram-positive microbes $B$. subtilis and S. aureus respectively, whereas the compounds SN4 and SN6 showed highest zone of inhibition (24 mm and $22 \mathrm{~mm})$ against gram- negative microbes $E$. coli and $P$. aeruginosa respectively.

Antifungal activity was also done by agar diffusion method. The investigation of antifungal screening data revealed that all the tested compounds are less active against $C$. albicans and moderately active against $A$. niger. The results were summarized in Table 3 . Compounds SN4, SN5 and SN6 showed moderate zone of inhibition (11, 10 and $11 \mathrm{~mm})$ against $C$. albicans respectively, whereas other compounds are less active. The compounds SN1 to SN7 showed a moderate activity against $A$. niger.

\section{Conclusion}

In a summary, we report the synthesis of ten novel 5-bromo-thiophene containing chalcone derivatives (SN1-SN10) by Claisen-Schmidt condensation under ultrasound conditions and conclude that the sonochemical reactions are simple to execute and the products are isolated in good yields when compared to the conventional method of synthesis. Also it was observed that, employing lithium hydroxide monohydrate $\left(\mathrm{LiOH}^{\mathrm{H}} \mathrm{H}_{2} \mathrm{O}\right)$ as a catalyst (base) provided the products in good yields after short reaction times with excellent chemoselectivity and purity under mild conditions. The antibacterial activity of thiophene chalcones was evaluated against some selected strains of gram-positive and gram-negative bacteria as well as fungal strains. Among them compounds SN4, SN6, SN7 and SN8 exhibited promising antibacterial activities whereas the same compounds had shown moderate to less active against fungal strains. In conclusion, the present study showed that the thiophene chalcone nucleus can be used as a template for future development through structure modification and to design more potent and selective antibacterial and antifungal agents.

\section{Acknowledgement}

The authors are grateful to M/s GITAM University for providing necessary facilities to carry out this research work. 


\section{References}

1. Romanelli G, Pasquale G, Sathicq A, Thomas H, Autino J and Vazquez P, $J$ Mol Catal A: Chem., 2011, 340(1-2), 24-32; DOI:10.1016/j.molcata.2011.03.004

2. Chtourou M, Abdelhedi R, Frikha M H and Trabelsi M, Ultrason Sonochem., 2010, 17(1), 246-249; DOI:10.1016/j.ultsonch.2009.06.008

3. Jarag K J, Pinjari D V, Pandit A B and Shankarling G S, Ultrason Sonochem., 2011, 18(2), 617-623; DOI:10.1016/j.ultsonch.2010.09.010

4. Srivastava S and Das B, Der Pharma Chemica, 2011, 3(6), 103-111.

5. Tomar V, Bhattacharjee G, Kamaluddin and Kumar A, Bioorg Med Chem Lett., 2007, 17(19), 5321-5324; DOI:10.1016/j.bmcl.2007.08.021

6. Bag S, Ramar S and Degani M S, Med Chem Res., 2009, 18(4), 309-316; DOI:10.1007/s00044-008-9128-x

7. Mandawad G G, Dawane B S, Beedkar S D, Khobragade C N and Yemul O S, Bioorg Med Chem., 2013, 21(1), 365-372; DOI:10.1016/j.bmc.2012.09.060

8. Hussain M M M, Bhat K I, Revanasiddappa B C, Siddiq A and Bharathi D R, Pharmacologyonline. 2011, 3, 880-888.

9. Romagnoli R, Baraldi P G, Carrion M D, Cara C L, Lopez O C, Preti D, Tolomeo M, Grimaudo S, Cristina A D, Zonta N, Balzarini J, Brancale A, Sarkare T and Hamel E, Bioorg Med Chem., 2008, 16(10), 5367-5376; DOI:10.1016/j.bmc.2008.04.026

10. Solomon V R and Lee H, Biomed Pharmacother., 2012, 66(3), 213-220; DOI:10.1016/j.biopha.2011.11.013

11. Rizvi S U F, Siddiqui H L, Nisar M, Khan N, and Khan I, Bioorg Med Chem Lett., 2012, 22(2), 942-944; DOI:10.1016/j.bmcl.2011.12.017

12. Kantevari S, Addla D, Bagul P K, Sridhar B and Banerjee S K, Bioorg Med Chem., 2011, 19(16), 4772-4781; DOI:10.1016/j.bmc.2011.06.085

13. Bhagat S, Sharma R, Sawant D M, Sharma L and Chakraborti A K, J Mol Catal A: Chem., 2006, 244(1-2), 20-24; DOI:10.1016/j.molcata.2005.08.039

14. Li J T, Yang W Z, Wang S X, Li S H, and Li T S, Ultrason Sonochem. 2002, 9(5), 237-239; DOI:10.1016/S1350-4177(02)00079-2

15. Chen G F, Jia H M, Zhang L Y, Chen B H and Li J T, Ultrason Sonochem., 2013, 20(2), 627-632; DOI:10.1016/j.ultsonch.2012.09.010

16. Colins $\mathrm{C} \mathrm{H}$ and Lyne $\mathrm{P} \mathrm{M}$, Microbial methods; $3^{\text {rd }}$ Ed., University Park Press, Baltimore, 1970.

17. Dixit R B, Vanparia S F, Patel T S, Jagani C L, Doshi H V and Dixit B C, Appl Organomet Chem., 2010, 24(5), 408-413; DOI:10.1002/aoc.1631 